\title{
Correction to: Gamma-Probe for Locating the Source of Ionizing Radiation
}

Timur Khabibullin, Andrey Starikovskiy, and Anastasia Tolstaya

\begin{abstract}
Correction to:
Chapter "Gamma-Probe for Locating the Source of Ionizing Radiation" in: A.V. Samsonovich and V.V. Klimov (eds.), Biologically Inspired Cognitive Architectures (BICA) for Young Scientists, Advances in Intelligent Systems and Computing 636, https://doi.org/10.1007/978-3-319-63940-6_37
\end{abstract}

\begin{abstract}
After publication of this chapter [Hecla J., Khabibullin T., Starikovskiy A., Tolstaya A. (2018) Gamma-Probe for Locating the Source of Ionizing Radiation. In: Samsonovich A., Klimov V. (eds) Biologically Inspired Cognitive Architectures (BICA) for Young Scientists. BICA 2017. Advances in Intelligent Systems and Computing, vol 636. Springer, Cham] it came to our attention that Jake Hecla was included as an author in error. The correct authorship is as follows: Timur Khabibullin, Andrey Starikovskiy, Anastasia Tolstaya. In addition, the affiliation of Timur Khabibullin, Andrey Starikovskiy and Anastasia Tolstaya was incorrectly shown. The correct affiliation of these authors is: National Research Nuclear University MEPhI (Moscow Engineering Physics Institute), Moscow, Russia.
\end{abstract}

The updated online version of this chapter can be found at https://doi.org/10.1007/978-3-319-63940-6_37 